\title{
Movement Pattern Recognition in Physical Rehabilitation - Cognitive Motivation-based IT Method and Algorithms
}

\author{
Veronika Szücs, Tibor Guzsvinecz, Attila Magyar \\ Department of Electrical Engineering and Information Systems, \\ University of Pannonia, Egyetem u. 10, 8200 Veszpém, Hungary \\ szucs@virt.uni-pannon.hu,guzsvinecz@virt.uni-pannon.hu, \\ magyar.attila@virt.uni-pannon.hu
}

\begin{abstract}
In this paper, a solution is presented to support both existing and future movement rehabilitation applications. The presented method combines the advantages of human-computer interaction-based movement therapy, with the cognitive property of intelligent decision-making systems. With this solution, therapy could be fully adapted to the needs of the patients and conditions while maintaining a sense of success in them, thereby motivating them. In our modern digital age, the development of HCI interfaces walks together with the growth of users' needs. The available technologies have limitations, which can reduce the effectiveness of modern input devices, such as the Kinect sensor or any other similar sensors. In this article, multiple newly developed and modified methods are introduced with the aim to overcome these limitations. These methods can fully adapt the movement pattern recognition to the users' skills. The main goals are to apply this method in movement rehabilitation, where the supervisor, a therapist can personalize the rehabilitation exercises due to the Distance Vector-based Gesture Recognition (DVGR), Reference Distance-based Synchronous/Asynchronous Movement Recognition (RDSMR/RDAMR) and the Real-Time Adaptive Movement Pattern Classification (RAMPC) methods.
\end{abstract}

Keywords: cognitive infocommunication; rehabilitation exercises; motivation; Kinect sensor; adaptive interface controller; real-time gesture recognition and classification

\section{Introduction}

The area of cognitive infocommunication is ready to highlight the new capabilities of ICT in human-machine interactions. The special features of the systems that develop cognitive abilities also reveal the added indirect ability that can help the efficient operation and widespread use of systems based on human-machine interaction $[1,2]$. 
Cognitive Infocommunication aims to create effective systems that are based on human perception, develop or return the cognitive ability of understanding and cognition to the user, all through models based on ICT engineering tools.

These include a number of studies that investigate the cognitive infocommunication environment, user experience, user experience [3], gesture recognition [4, 5] eye-tracking [6], BCIs [7, 8], VRs [9, 10], virtual lab [11, 12, 13], serious games, gamification [14] and other educational environments [15, 16]. These research findings are of great importance in the field of education, development, rehabilitation or even the industrial application of robotics.

One of the most important areas of application concerned is the field of healthcare information technology, including motion rehabilitation.

Unfortunately, stroke became one of the most frequent diseases of the modern day. All over the world and unfortunately also in Hungary, stroke is the most frequent cause of death and disabilities. Stroke has become the third-fifth cause of death: Every 20 deaths is caused by stroke, meaning that in America 140000 people are killed by stroke each year. Every year, 795000 people have a stroke and, out of them, 610000 is a new stroke victim. Stroke is not just the disease of the elderly, but unfortunately, in recent years it is increasingly affecting young people. The number of patients increases year by year. In Hungary, 42000 people suffer a stroke every year and 13000 people die of brain damage. Twenty-five percent of patients are younger than 60 years of age, but men have a stroke five years earlier than women on average and this has several social consequences [17]. Those who manage to survive the disease are also seriously threatened, as nearly 24 percent in the first year, and another 5 percent lose their lives in the second year. The stroke may affect one's life. According to statistical data, 48 percent of brain-to-asthma survivors suffer from half-side paralysis, but some degree of cognitive decline can be detected in more than 60 percent of cases. 22 percent of patients are impotent, many of whom are forced to a wheelchair. 24-53 percent of those who have undergone stroke are partially or completely dependent on others, 12-18 percent are aphasic (speech disorder) and 32 percent of them are depressed. This makes it necessary to involve devices of modern technology [18, 19].

Besides this, nowadays, the development of virtual reality (VR) applications is one of the most dynamically developing areas in the field of information technology. In the case of movement rehabilitation applications, modern sensors can be used as input devices. These sensors are of great importance, mainly those that are operating based on optical principles because of their convenient usability. $[20,21]$ The role of these IT solutions, serious games and frameworks that can be used in home environments is also significant in post-stroke rehabilitation. Many of these have been developed worldwide in the past years. Sadly, not many of them became popular as they did not achieve the hope people placed in them. The main cause of these unsuccessful rehabilitation projects was clear from experience 
gained in previous projects: the use of IT equipment that is difficult for patients, supporting people, nurses and also the loss of motivation of patients [22].

Depression of stroke patients often slows down the recovery process. Karaahmet et al. [23] examined the risk factors for the occurrence of this phenomenon. In their study, they found out that based on both post-stroke state and the improvement monitoring FIM test, that the improvement of patients with depression is significantly slower. Because of this and consequently, a lack of motivation appears as a generator and/or as a consequence of depression. The combined effect of these two can most likely cause the failure of rehabilitation [24, 25].

Rehabilitation can be made easier for patients as well, if rehabilitation exercises, gestures can be performed in an interesting modern environment. There are many computer-aided solutions already available that help with traditional therapy: the patient must have direct contact with the said computer (pressing keys, using a mouse) which can be a difficult task. Bruno and his colleagues have studied the possibilities of using commercial video games in rehabilitation in a study starting in 2015. Their study points out that based on 4728 related articles and abstracts of which 547 are focused on post-stroke rehabilitation and movement rehabilitation, video games could achieve similar results to conventional therapies [26, 27].

Motion tracking devices that have appeared in the last decade allow the user to not have physical contact with the control device, thus these devices make it easier to control difficult serious games. The use of devices with motion detection sensors could be useful in rehabilitation software. For example, the Leap Motion gesture sensor, the V1 (Xbox 360) and V2 (Xbox One) versions of the Kinect sensor and the Shimmer sensor as well. When using the Kinect sensor, various problems have arisen, for example, the difficulty of controlling as the device does not always perceive well or the gestures are inaccurately identified [28, 29]. The examination of these problems is mandatory because if there is no solution for them, then, unfortunately, it will be impossible to effectively use serious games or software for rehabilitation purposes [30, 31, 32]. Worthen et al. and Llorens et al. created multiple recommendations for exercise games (exergames) with the aim of stroke rehabilitation. With these recommendations, it is possible to deduce that the need for serious games exists for both therapists and patients. However, the requirements of their usability are not always fully met, therefore, the developments will not be applied to the caring protocols after the initial testing.

In 2017, Afyouni et al. presented a framework for stroke rehabilitation which gives a possible solution to the adaptive deficiencies stated by Worthen et al. and Llorens et al. In their framework, Afyouni et al. adapted the difficulty of the tasks during multiple cycles to the current state of the user [33].

However, the fact is that the exergames could not adapt to the needs of the patients if the correction values are pre-set or the therapist calibrated the application beforehand [34]. 
In this paper an adapting solution to the mentioned problem is presented: When using gesture recognition and evaluating the gestures in an application controlled with the Kinect sensor, the algorithm is capable to adapt to the state of the user in real-time.

The goal of the algorithm is to recognize gesture patterns during movement rehabilitation while classifying and evaluating the gesture patterns. This is an expectation regarding the basis of the therapy when using a software method.

One of the critical points of motion-based application control is the retrieval of motion descriptors from the sensor used as the input interface throughout the application. The present work of the authors was designed to extract, to process, analyze and to improve the motion data of the Microsoft Kinect sensor. The set of function sets provided by the sensor manufacturers to the developers allows the data to be accessed, but this alone is not enough for the developers and users of rehabilitation applications. There is too much uncertainty for the user. For example, the loss of connection during practice or a series of unsuccessful tries can demotivate the user.

\section{Data Acquisition and Data Analysis}

\subsection{Data Acquisition}

If the appropriate methodology presented in the next chapter is used, the loss of motivation can be avoided. More importantly, Kinect applications often appear in the field of movement rehabilitation, like the tools related to telemedicine systems. This means that the personal presence of the therapist is not required as telemonitoring does not necessarily take place in real-time, but nevertheless, it can provide the medical staff with therapeutic process tracking and evaluation of the results in a very human-like, graphical way.

Data logging is one of the basic tools that allow performing the tasks mentioned in the problem discussion. There are two versions of the sensors, the former Kinect for Xbox 360 sensor and the new Kinect for Xbox One.

In the data logger application, all or only the selected joint points are logged at the 30 fps sampling rate of the device, and every single repetitive movement can be described with 67 frames on average. Instead of the time-stamp provided by the device, the more efficiently used ratio number is stored during the skimming of motion descriptors, that are calculated from the time-stamp in milliseconds, based on the elapsed time from the start of the motion. 


\subsection{Data Conditioning and Analysis}

By processing the stored data, a statistical analysis of movement can be provided and also it is possible to reconstruct from descriptive data.

The available data is noisy $[28,29]$ which is partly due to the random error on the data or the values calculated by the sensor instead of the measured value. These values are generally outliers that are largely different from the correct data. The measurement result, the subsequent processing, the accuracy of the gesture recognition can be influenced by the noise on the measured signal or the measurement error meaning that the signal must be filtered and made error-free before processing.

Of course, the goal is to be able to perform the processing in real-time, not just when processing the stored data. To do this, it is necessary to examine the running time of data clearing performance and to improve the accuracy of gesture recognition after the filtering.

Initially, several error correction algorithms were used, like 7th, 9th polynomials and Lagrange interpolation was also applied to the original data set.

\subsubsection{Filtering with Lagrange Interpolation}

If there is a set of $\mathrm{N}$ points on a Cartesian plane, there will always exist an $\mathrm{N}-1$ th order polynomial of the form

$$
\mathrm{y}=\mathrm{a}_{0}+\mathrm{a}_{1} \mathrm{x}+\mathrm{a}_{2} \mathrm{x}^{2}+\ldots+\mathrm{a}_{\mathrm{n}-1} \mathrm{x}^{\mathrm{n}-1}
$$

which passes through all the points. Lagrange interpolation came up with a neat approach to finding this polynomial, which constructs a set of 'basis' polynomials which are zero at all specified points except for one, then scale and add them to match all control points.

When using Lagrange interpolation, the polynomial of the lowest possible number is used. Unfortunately, it is distorted at interval endpoints. The approximation method gave much worse data than the original measured data. To resolve this problem, another method was used which was a windowed interpolation technique. This interpolated through 10 measurement frame-windows by sliding the window until the full data set was processed. The comparison of the resulting data set and the original motion descriptors, then averaging the descriptor of the movement, gave a data set that was free from abnormal measurements and abnormal values.

Unfortunately, with the Lagrange interpolation approach, it took a very long time to process the input data as it was experienced, so a different polynomial fitting was used which gave very good results. 


\subsubsection{Filtering with Polyfit Function in Matlab}

The data set was filtered using Matlab's built-in polyfit.m function in this case. When using the function, a predetermined degree of polynomials are available to be fitted. The 9th polynomial matching has the best results in our case.

It's syntax:

$$
p=\operatorname{polyfit}(x, y, n)
$$

$\mathrm{p}$ returns the coefficients of the polynomial that fits best (examining the deviation of the least-squares differences) to the data of $y$.

$$
p(x)=p_{1} x^{n}+p_{2} x^{n-1}+\ldots+p_{n} x+p_{n-1}
$$

the results of this were used in the following parts.

Before doing the aforementioned filtering, it should be noted that the measurement data, like all measurements, can contain measurement errors that arise from environmental factors, such as the IR range of room-induced solar radiation can interfere with the sensor, or the frequency of the ignition transformer of the neon-tube lighting in the room can cause sensory disturbance. Changing the light conditions in the room used for measurement may impair the stability of the sensing.

These errors should be separated from the faults caused by the uncertainty of the movements of the user. These faults can be considered as errors or as faulty gestures from the point of view of the therapy.

The user's gesture can always be matched to a predefined gesture pattern. However, if it contains data that is significantly different from the sample to the extent that the gesture descriptor cannot be transformed near the sample descriptive values by simple filtering, it may be a defective gesture and not a defective descriptor resulting from the measurement.

\subsubsection{Constructing the Error-Free Result Matrix}

After calculating the polynomial data for the new curve, the resulting matrix was compared with the matrix containing the original data set of measurements at the same index locations. The difference was formed for each index and that was stored in $\mathbf{t}_{\mathbf{k}}$ variables.

$$
t_{k}=\left|m_{k}-y_{k}\right|
$$

The mean distance a has been calculated:

$$
\mathrm{a}=\frac{\sum_{k=1}^{n} \mathrm{t}_{\mathrm{k}}}{\mathrm{k}}
$$


As the next step, the data set containing the original measurement data set and the polynomial curve data was re-examined in pairs.

In the movement descriptor matrices the absolute value of the deviation of the values at the same index positions has been compared with the average deviation previously calculated. If this deviation was smaller than the calculated average deviation, then the original measurement data was considered good and remained in place. If it was higher than average, then the original data was considered and replaced with the replacement polynomial at the given position in the matrix with the following formula:

$$
u_{k}=\left\{\begin{array}{l}
\text { if } t_{k}<a \text { then } m_{k} \\
\text { if } t_{k}>a \text { then } y_{k}
\end{array}\right.
$$

The next section introduces the algorithms that were developed to improve the effectiveness and accuracy of pattern recognition.

\subsection{Developed Movement Pattern Recognition and Classification Methods}

\subsubsection{Reconstruction of Movement Patterns/Gestures}

In virtual and augmented reality applications, the most important for the human viewer is to see how deeply they can experience the effects of the perceived environment.

As already mentioned in the earlier sections, the optical sensors are popular with movement rehabilitation applications. In this study, the main focus was on the Kinect sensor.

The Microsoft Kinect sensor uses a built-in infrared depth emitter and camera, while it also uses a four-microphone array to detect the location of the user inside a closed space. The sensor works with a randomized decision forest. In addition, it uses the mean-shift clustering algorithm to determine which pixel belongs to which body part.

There are many cases in the physical rehabilitation process where certain serious game elements are incorporated to maintain motivation. Similar or repetitive gestures can be used to practice or to control a game.

Due to sensory error, movement recognition is often not eligible as the game could think that the gesture is incorrect. This results in the loss of motivation of the patient/player. The possibilities for more precise recognition of gestures will be introduced in the following chapter.

Two different methods for recognizing movement patterns are presented in this paper. Both of these methods can be applied in the time-dependent or time- 
independent mode for movement (gesture) recognition. The methods are reference-based methods, one of them is a reference-based time dependent and time independent distance vector differentiation:

Distance Vector Based Gesture Recognition (DVGR) method, while the other is based on a fixed-distance correlation of joints: Reference Distance Based Synchronous/Asynchronous Movement Recognition (RDSMR/RDAMR) methods.

\subsubsection{Reference-based Recognition and Identification of Movement Patterns}

The two methods in general:

A gesture descriptor base functions as a basis for analysis for both mentioned gesture pattern processing methods. In both cases, the reference movement patterns are gathered beforehand, then during the processing and the recognition phases, the algorithm compares the actual movement pattern data descriptors to the base data in real-time.

Gesture analysis:

Analyzing gestures is a complex sequence. Its main functions are that it analyzes the gestures of a human, and decides which movement it is $[35,36]$.

The problem consists of multiple layers and is a difficult task. The primary problem is recognizing a human or shape on the picture. The previous methods could only recognize shapes in colored, one-dimensional pictures or in monochrome ones.

Its sequence is the following:

- The first step is determining the background and saving it as a starting basis.

- After determining the background, in the second step, the algorithm watches whether change happens in the picture. The simplest method was to compare the original and the newly transformed, black and white transformed new picture: If there are differences, then the pixels differ in the image.

- During the third step, the difference and the shape in the picture are determined. The usable technique is, e.g. a circle delimit method, where the deviation is delimited into a circle with a polygon and by analyzing this polygon the shape is defined

Defining the shape in itself is not enough for gesture pattern recognition. Other tasks include recognizing the gesture and its tracking. For doing such tasks, methods will be presented in the next sections. The basis of both methods is that it tries to recognize a saved gesture pattern which was gathered beforehand by using real-time data from the user. The user who does the movement sees a video during 
the gesture and has to follow it as precisely as possible. To understand and to recognize the gesture pattern, the identification of the user is not essential, only the skeleton data provided by the Kinect sensor is used instead of the picture data.

In these types of movement recognition, after the required movement pattern identification, the therapist teaches the reference motions, teaches the exercises to the algorithm - in other words records the references inside the application. In this reference movement recording state, the video file has to be stored and of course, the movement descriptors are stored together with the records. During these types of movement recognition, several different gestures and movement samples are stored in the application.

The job of the patients is to complete the tasks prescribed for practice as accurately as possible.

Movement detection with the Kinect sensor takes place in real-time in a threedimensional space using $x, y, z$ coordinates measured in meters away from the device. A simple gesture is about 60-70 frames on average, but depending on the complexity of the given gesture, the number of frames may be more. The essence of gesture recognition is that the processor continuously compares the coordinate triplets describing the movement of the patient to all enabled reference gestures in each frame.

The job of the application is to monitor which of the references has found a match with which frame/sequence and give feedback to the user.

However, in the early stages of rehabilitation, the patient's movement coordination is so weak that his gestures are mostly incorrect. In these cases, the recognizing software does not evaluate the gestures and does not give feedback to the user to avoid demotivation.

Expectations from the gesture processing methods (Fig. 1):

The methods, algorithms of gesture pattern analysis have to fit the following minimal expectations:

- It should be used when dealing with the practice of gestures that are not defined beforehand, meaning the application should accept more than one gesture including ones that are personalized or made by the users.

- Real-time feedback: During practice, the application has to give real-time feedback after each repetition. The feedback has to consist of movement acceptation or refusal. Also, this feedback must not slow the processing application. 


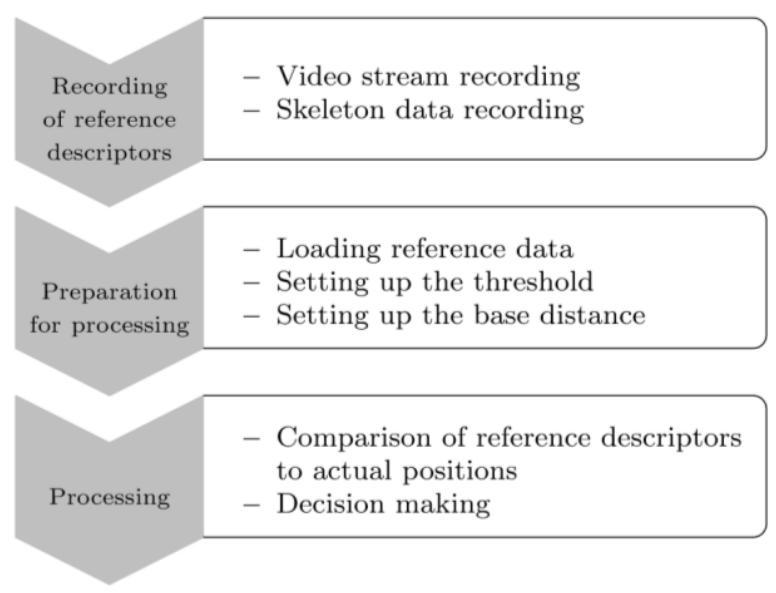

Figure 1

General method to process movement descriptors

\subsubsection{DSMR/RDAMR - Reference Distance-based Synchronous / Asynchronous Movement Recognition Method}

The other method of movement detection is synchronous/ asynchronous analysis, which is also based on a reference gesture base. However, in this case, the coordinates describing the movement of the user are calculated in a way that takes the base shift into account. The base shift characterizes the current position of the user relative to the reference movement when it was registered, in other words, it assumes a basic distance as a constant (Fig. 2). During gesture identification, the algorithm monitors the distance between the joint points of the skeleton, corrected by the base distance constant which determines which reference pattern can be applied with the highest precision.

In the case of synchronous analysis, with the time elapsed between successive frames of the movement pattern, the speed of the gesture could be calculated. In the initial rehabilitation phase, this expectation of the patients is too much. Therefore, the other method, the asynchronous analysis is used by this application, meaning that it only takes the coordinate triplets into account which can be found in each movement descriptor frame. The elapsed time is not taken into account which means that the speed of the exercises does not affect the qualification of the gesture.

In serious games, similar to movement pattern exercises, simple, often-repeated actions are performed by patients to control the game. Successful game controlling (or successful practice) is a requirement to allow the sensor to identify the gestures in real-time and to allow the software to adapt to the current abilities of the patients. This way, they are motivated for further exercise. 


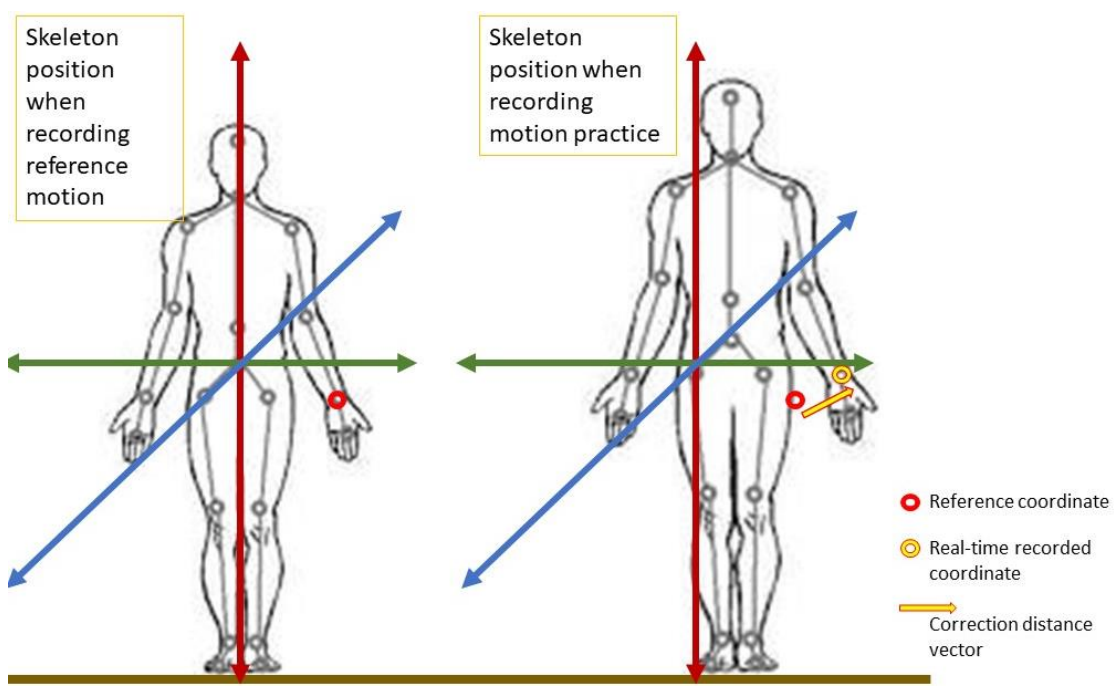

Figure 2

Baseline distance model

The application monitors which of the references has found a match with which frame-sequence. It returns feedback after identifying the matching gesture. This is an important part of the process, many previous studies have shown that positive feedback motivates patients.

However, in the early stages of rehabilitation, the patient's movement coordination is so weak that his gestures are mostly incorrect. In these cases, the recognizing software does not evaluate the gestures and does not give feedback to the user to avoid demotivation.

The formal diagram of RDSMR/RDAMR methods can be seen in Figure 3.

The main difference between asynchronous and synchronous movement recognition method is the speed of the analyzed movement.

The main advantage of the proposed method that it can be used in timeindependent mode, because the evaluation process calculates only with the sequence of movement descriptors, but the result does not depend on the elapsed time between two consecutive descriptors. 


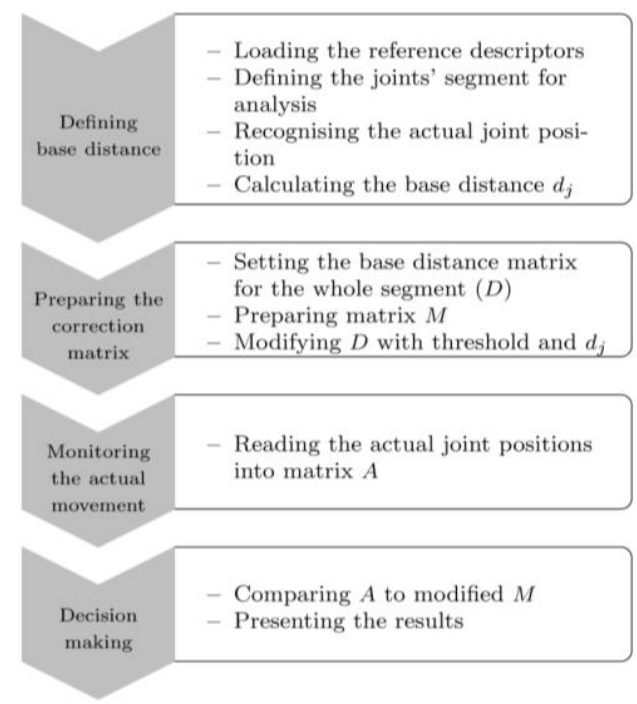

Figure 3

Baseline distance method

\subsubsection{DVGR - Distance Vector-based Gesture Recognition Method}

During the research, the Kinect v1 sensor has been used which can track 20 joints in a skeleton.

The problem is that when the user stands at a different distance from the sensor than when recording the reference movement, the sensor sees different $\mathrm{X}, \mathrm{Y}, \mathrm{Z}$ coordinate positions of the joints. This could lead to problems when processing data.

This could be solved by using the distance vectors between two joints. These vectors could provide the basis of comparison in the future.

Aside from the assigned joints from the side of the body, another joint is selected which has a pseudo-static attribute regarding movement, which is called a base joint. This base joint is compared with a distance vector to the position-triplets of other, analyzed joints when using the DVGR method. For example, when analyzing a hand movement, the left-hand descriptors are made by subtracting the analyzed wrist and elbow coordinates from the coordinates of the left shoulder. These distance vectors provide a momentary position.

Figure 4 presents the formal working progress of the DVGR -Distance Vector Based Gesture Recognition method. 


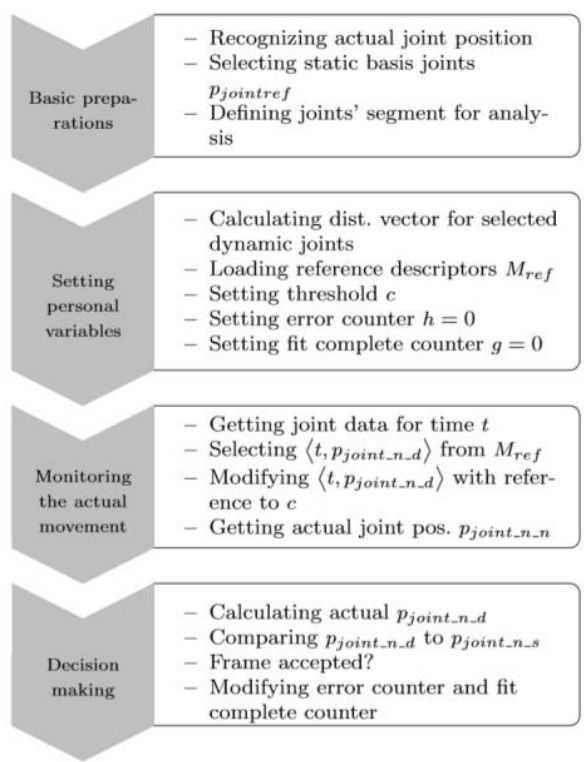

Figure 4

Distance Vector-based Gesture Recognition method

\section{Notations:}

- $\mathrm{t}$ is the actual loaded sample/measured time index,

- s means recorded reference (sample) data,

- a means the actual measured position data,

- joint means the selected joint from the analyzed joints,

- $\mathrm{n}$ is the number of joints in the selected segment,

$-\mathrm{x}, \mathrm{y}, \mathrm{z}$ are the Cartesian coordinates for each joint,

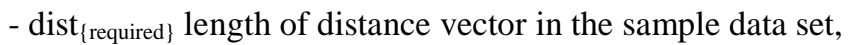

- dist $_{\{\text {real }\}}$ is the length of distance vector for the real joint position

- Static reference joint is $\mathrm{p}_{\left.\{\text {joint_ref\} }\} \mathrm{x}_{-}\{\mathrm{ref}\}, \mathrm{y}_{-}\{\mathrm{ref}\}, \mathrm{z}_{-}\{\mathrm{ref}\}\right)}$, measured position

- The actual measured joint position is $\mathrm{p}_{\{\mathrm{j} \text { oint_n } \mathrm{n}\}(\mathrm{xt}, \mathrm{yt}, \mathrm{zt})\}}$

- $\mathrm{c}$ is a threshold variable, which gives the possibility to personalize the method, input variable for the algorithm

- $\mathrm{h}$ is a counter for not fitting frames

- $\mathrm{g}$ is a counter for fitting frames 


\subsection{Gesture Classification - Real-Time Adaptive Movement Pattern Classification (RAMPC) Method}

The most important information for classification is whether the gesture for the given control was appropriate, acceptable or not. Successful classification required an additional method, its principle is shown in Figure 5.

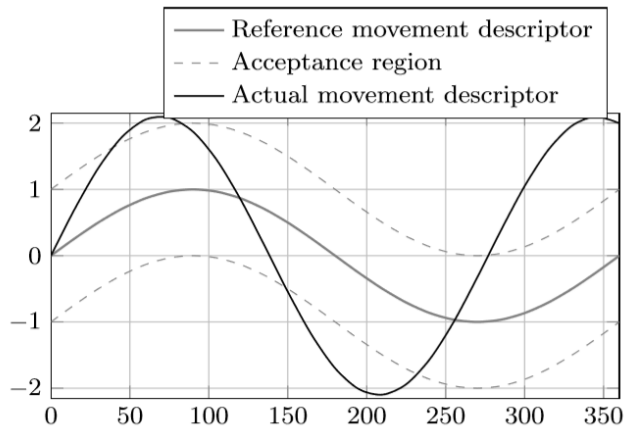

Figure 5

Classification

The classification works with a reference movement descriptor.

The algorithm allows for using multiple saved reference movement descriptors. Each different gesture descriptor can be found in a personal, holding vector. If the hand of the user is in the correct starting position in the correct time/frame (few tenths of seconds), also counting the threshold, then the application increases the index of the vector. However, the application can increment the index in multiple vectors as multiple gestures can start from the same position. If no index has been increased in the frame, then the algorithm increases the number of errors. If the number of errors reaches a predetermined value, then every index will be set to zero and the gesture recognition will start from the beginning.

The counter can be modified in an inverse direction as well. This happens when the user correctly does the movement in the frame and the application counts that as a correct frame by adding it to its respective vector as each vector has its own correct frame counter. Naturally, the done movements cannot be fully the same as they were saved beforehand. Therefore, if the user fails the last 10-20 indices of the movements (depends on the threshold value), but their error number is below a predetermined value, then the gesture is accepted.

The user can add gestures to the application, but the gestures do not have to be done in the saved order. Every sequence can be accepted and the movements can not only be done once but multiple times. 
For example, look at a circular hand movement. The 3D domain of the reference movement is expanded: both in positive and in negative directions. A broader tolerance threshold is defined around the circular movement from the outside and from the inside. This range will be the acceptance range later on and serves as a basis for classification. The other standpoint which must correspond to the motion-descriptive coordinate set is that the number of motion-describing frames for a movement pattern averages $60-70$ frames. Taking the faulty measurements and deviations due to incorrect movements of the user into account, the descriptive frames must be within the acceptance range in a predetermined amount of $501 \%$ by default. During therapy and exercises, the goal is to have as many coordinates of the gesture as possible in this acceptance range. If the coordinate triplet of the frame is outside, then it cannot be considered part of the expected gesture. Like the previous sections, there are also reference gestures, reference descriptors in this case, but they are not stored on the basis of an external sample. Instead, they are calculated from the average of the first few movements of the given patient and are there completely adaptive to the current user and their capabilities.

Classification can be done with any periodically repeating gesture. They do not have to necessarily be circular movements.

This classification algorithm ensures that the qualification, acceptance or rejection of the movements is always adapted to the needs of the patients.

The main algorithmic steps of the classifications can be seen in Fig. 6 .

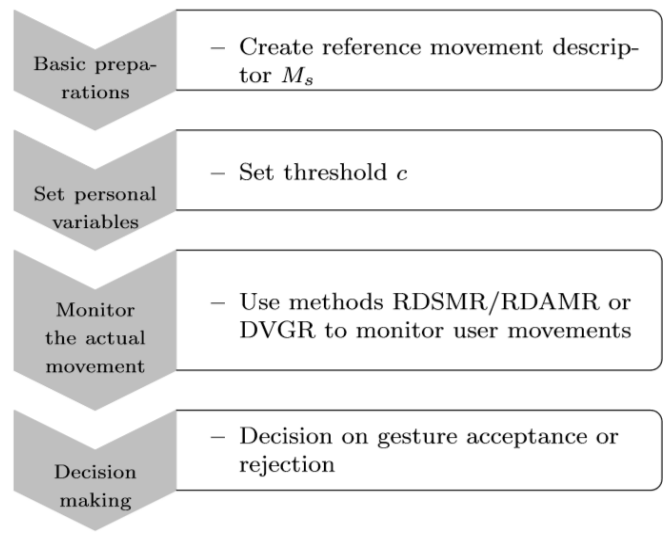

Figure 6

Basic classification method

A question arises: is the symmetric acceptance domain useful, or different ranges should be defined for different directions? The next section gives proof of this because by using the adaptive acceptance domain for every classification step that could be adapted to the user's (patient's) own capabilities, it will automatically adapt to the progression of the user. 


\subsection{Adaptive Acceptance Domain}

During classification, one of the most important aspects is that the application should work in real-time, observe the movement of the user and evaluate them with the shortest delay and response time possible. Following this approach, an adaptive control application has been developed to support applications using the Microsoft Kinect sensor in real-time and it uses the classification algorithm presented above. During its operation, it monitors the gestures of the user in realtime and compares the reference gestures generated from the first gestures. It also takes the acceptance range into account which in the present case is a distance of $d$ meters in positive and negative directions, so its scale is always a symmetric interval of $\mathbf{2 d}$.

By default, if more than $50 \%$ of the frame of the gesture is within the range of $\pm d$ of the reference value, the gesture is accepted. Also, the therapist could increase or decrease the range of $d$ to adjust to the rehabilitation program of the patient. The acceptance rate of $5 \%$ can also be increased or decreased, resulting in a fully customized therapy.

As with the simple recognition presented earlier, the users also receive positive feedback if the gesture is successful to motivate them to continue their practice.

The next session introduces a case study, where the authors performed a user test to give the proof of concept.

\section{Experimental Results - Case Study}

\subsection{Record Movement Descriptors}

To prove the initial condition, the first step is to record large amounts of data. This process required 15 people who performed different movements, gestures. The data were recorded on the basis of the practice of physiotherapists in stroke rehabilitation. All movements are a typical SRM (Single Repetitive Movement) movement. SRM means that in rehabilitation, all patients have to repeat the movements 15-50 times, sometimes even 100 times or have to repeat them continuously for $2-5$ minutes.

With the simultaneous launch of the two sensors, data logging software allows motion-based files to be recorded in text file format. The main advantage of the recorded files is that these are easy to process.

The scenario of data capturing: 
Data capturing consists of two parts. In the first one, six different gesture exercises have to be done during the synchronous operation of both sensors. These exercises mainly concentrate on upper limb movements. The tester was put $180 \mathrm{~cm}$ away from the sensors as that was the distance which was the most appropriate for the authors.

Establishing the exercises:

- $1^{\text {st }}$ measurement: The tester, with their palms toward the sensor at shoulder height, starts to move both their hands in circular motion ten times.

- $\quad 2^{\text {nd }}$ measurement: Next, the hands have to be extended to the side, then retracted ten times while the palms are facing the sensor.

- $\quad 3^{\text {rd }}$ measurement: At the start, the arms are at ease next to the body. Then, they have to be raised to shoulder height, extending sideways and in the end, going back to the starting position. The repetition number is also ten.

- $4^{\text {th }}$ measurement: The arms start in the same position as in the $3^{\text {rd }}$ measurement. Then, they have to be raised over the head while they are extended sideways, repeating ten times.

- $5^{\text {th }}$ measurement: At the start, the legs of the user are closed. First, the right, then the left leg has to be raised sideways in 45-60 degrees, repeating five times.

- $6-7^{\text {th }}$ measurements: The tester sits on a chair, where their arms are raised to shoulder height and their palms are facing the sensor. The tester raises both their arms above their head, then back down, repeating five times. The arms only move up or down as sideways movements are prohibited. During this, both their legs are raised, extended then put down to the ground, repeating five times.

Recorded files contain a matrix of coordinate-triplets: in every row, the matrix starts with a time-stamp, and the joints' coordinates follow. For each cycle, there are 60 movement descriptors stored.

After recording movement data, it was necessary to analyze and decide, if filtering and smoothing of data were needed or not.

During processing, the data set of the measurement data (i.e. the patient movement) has been examined. The first purpose of the study was to find out whether an error-correcting algorithm that supports movement detection in the later phase of processing would be needed. The second purpose was to find out if that algorithm would allow more accuracy during detection. 


\subsection{Filtering and/or Polynomial Fitting for an Error-Free Data Set}

By comparing the original set of data and the error-free data set, the modified index location can be determined which also reveals which original data was incorrect.

After the data smoothing, the cycle searching process was performed on the data. It should be considered, that the found difference between the repetitions on the original data and on the smoothed data was only 5-15\%. Meaning that real-time processing cannot be used because of the long processing time, but the result of the original data set is not usable if every 5th movement was recognized as a failed movement. This result indicates to use an adaptive acceptance domain for classifying the recorded movements.

\subsection{Reconstruction of Movement Patterns}

After the measurement data is input into the Matlab/Scilab/Excel environment, where the reconstruction of the movement pattern can be done 1D, 2D and 3D while taking the elapsed time into account.

During the reconstruction, the viewer receives a real picture of the nature of the movements performed during the therapeutic practice, the number of repetitions, and the graphical display. For example, deviation from a reference motion pattern.
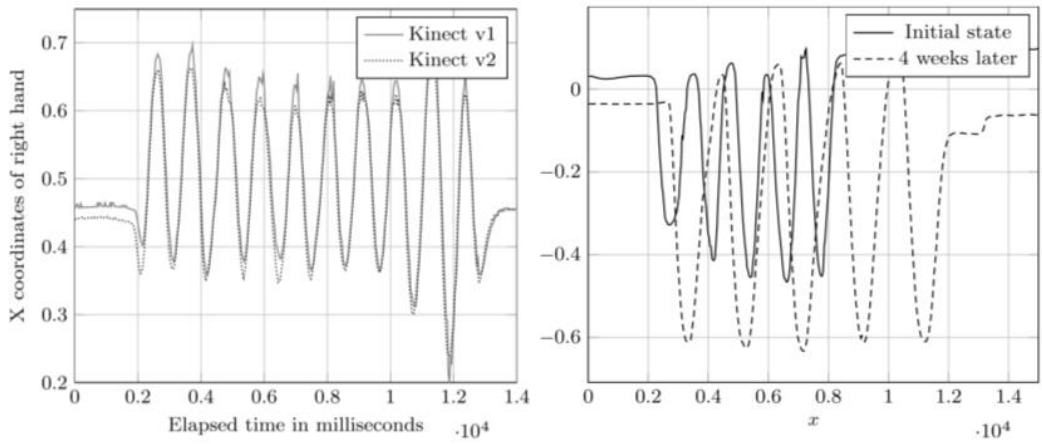

Figure $7 \mathrm{a}, \mathrm{b}$

Circular movement with the Kinect v1 and v2 sensors, X coordinates and elapsed time; b) Leg raises in different periods of rehabilitation, $\mathrm{X}$ coordinates and the elapsed time

As can be seen in the reconstruction figures Figure $7 \mathrm{a}$ and Figure $7 \mathrm{~b}$, it can be decided by the therapist looking at the measurement data set that whether there is a progress in the movement rehabilitation of the patient or not. 


\subsection{Classification and Transform Movement Data into the Adaptive Acceptance Domain}

To keep the motivation for further exercises, it is necessary to ensure the patient's continuous successful experience, a movement assessment that adapts to their special needs. The different acceptance domains can be seen in Figures 8-9.

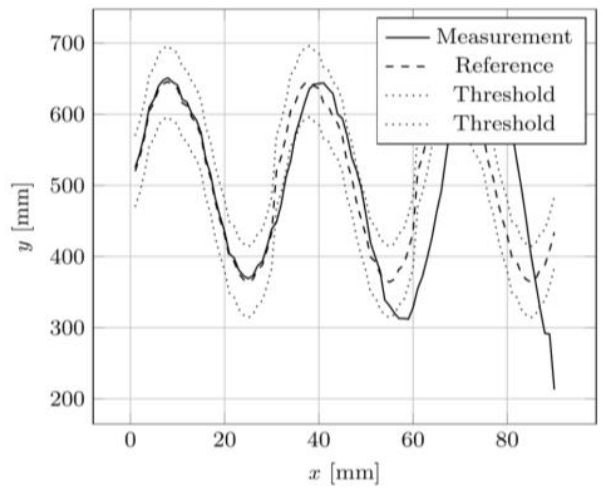

Figure 8

The acceptance domain is $\mathbf{\$ 5 0} \mathrm{mm}$ over the reference movement descriptors
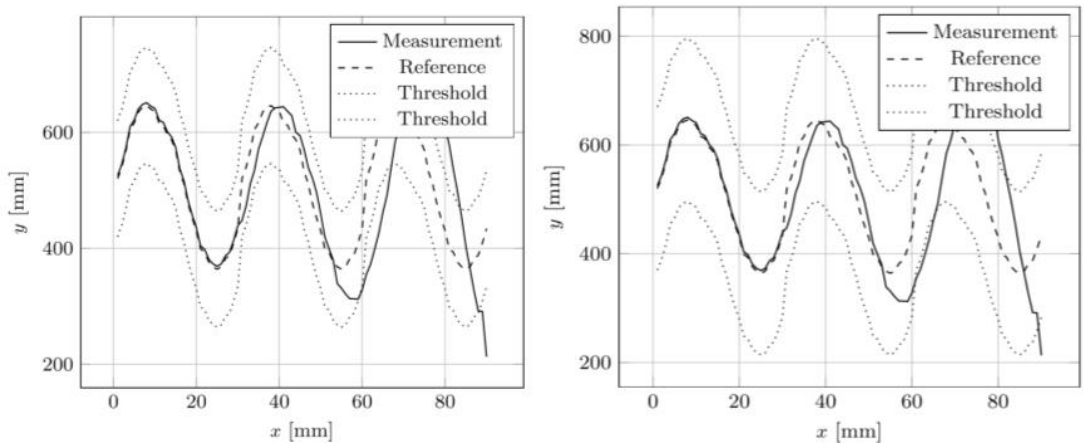

Figure 9 a,b

a) The acceptance domain is $\pm 100 \mathrm{~mm}$ over the reference movement descriptors; b) The acceptance domain is $\pm 150 \mathrm{~mm}$ over the reference movement descriptors

As can be seen in Figure 9b, where the acceptance domain size was $\pm 150 \mathrm{~mm}$ over the reference movement and the acceptance ratio was $50 \%$ of contained frames, the first whole movement cycle was fitted in $96 \%$ and was accepted. The second movement cycle was fitted in 53\% and also was accepted, but the third movement cycle was fitted in $37 \%$ and it was refused.

If the therapist chooses other acceptance rate, such as, $60 \%$, it means, that the second cycle could be refused because of the more strict classification parameter. This method can be applied in the developing state of the patients. 
In Figure 9, the different acceptance range can be seen. In this situation the acceptance range is $\pm 100 \mathrm{~mm}$ over the reference movement, the acceptance ratio is $50 \%$. As is presented in Figure 9, the third movement cycle was refused on a $33 \%$ fitting ratio, as in the previous sample, but using $\pm 50 \mathrm{~mm}$ acceptance range over the reference movement Figure 8, it will be refused on a $16 \%$ fitting ratio.

These samples show that the size of the acceptance domain, together with the fitting ratio, on every movement cycle can give a solution to accept purer and unstable movements, when the user could not make more precise gestures, and it could result higher motivation, more initiative on the part of the user to continue the exercises.

\subsection{Discussion of the Results}

After capturing the motion data, the authors investigated whether the time needed to produce the corrected data set was suitable for real-time processing or not, as well as whether there was a significant difference after correction in the number of recognized, correctly identified cycles or not. Based on the results, the Lagrange interpolation data filter takes too much time, it is not suitable for real-time movement detection. The polynomial fitting method allows the data to be filtered during runtime but does not bring significant improvements to the recognized movement cycles. This means that it is not advisable to perform pre-filtering and data correction before using the movement detection algorithm.

During recognizing movement patterns, the best result is achieved by the distance vector-based pattern matching which is also the primary test method for classification using the data-accepting range.

Finally, it can be concluded that the acceptance range-based adaptive movement classification for Kinect can be used in real-time. This method provides effective support for adapting to the patient's current capabilities throughout the rehabilitation process, despite the coordination constraints of motion while always ensuring successful software utilization, maintaining motivation and preserving the gaming experience which is one of the pillars of effective rehabilitation.

\section{Conclusions}

The analysis of motion data, pattern recognition, reconstruction and visualization of motion patterns are the basic questions of the personalization of movement rehabilitation applications, which indirectly support the effective use of IT tools.

Inadequate interaction tools, especially in mobility rehabilitation applications endanger the long-term use of the applications as the user becomes unmotivated and their interest is lost while also endangering the positive outcomes and efficacy of the treatments. 
The author's experience gained in the international and national projects confirms that the continuous success and motivation of the user is an important condition for successful treatment.

There is a need to develop a method that provides a sense of success for the user while using the rehabilitation applications with the Kinect sensor in a way that gestures are processed and categorized in real-time and adapted to the special needs of the user.

The motion data extracted from the Kinect sensor contains estimated values when the sensor loses contact with the user and also contains misaligned coordinate data. In the course of the investigations, the result was that the preliminary error correction of the data is not necessary during the subsequent processing: Errors during the recognition and classification of the gestures do not result in significant deviations in negative and in positive directions.

The studies carried out showed that in the case of the Kinect sensor, the different processing methods of the motion data associated with the 'skeleton' showed different results in the recognition of the gestures.

Methods that were developed simultaneously with the research, the DVGR and RDSMR/RDAMR methods can be used to identify users' therapeutic gestures used in rehabilitation applications in real-time with high accuracy in the application control.

The Real-Time Acceptance Range-based Adaptive Movement Pattern Model Classification (RAMPC) supports Kinect sensor's control of motion-recovery gaming applications, which according to the user's needs, defines - based on relative reference base generation - controllable parameters for a dynamic acceptance domain to accept user gestures. This method makes Kinect sensorydriven motion rehabilitation applications applicable at every stage of the therapy, so it does not cause any unsuccessful movements during exercise, does not cause a demotivating effect in a high degree of motion limitation, and therefore, is likely to maintain a constant motivation for the user.

\section{Acknowledgement}

The authors would like to thank every colleague who helped their work during testing, to Ms. Petra Marton for her contribution in the data logger application development, to Ms. Mónika Ozsváth for her contribution in the development of data conditioning and reconstruction.

The Authors acknowledge the financial support of Széchenyi 2020 under the EFOP-3.6.1-16-2016-00015.

A. Magyar was supported by the János Bolyai Research Scholarship of the Hungarian Academy of Sciences. A. Magyar was supported by the UNKP-19-4 new national excellence program of the Ministry for Innovation and Technology. 


\section{References}

[1] Baranyi, P., Csapo, A., \& Sallai, G.: Cognitive Infocommunications (CogInfoCom) Springer, 2015

[2] Gyula Sallai: The Cradle of Cognitive Infocommunications. Acta Polytechnica Hungarica, Vol. 9, No. 1, 2012, pp. 171-181

[3] Balint Szabo, Karoly Hercegfi: Research questions on integrating user experience approaches into software development processes. $7^{\text {th }}$ IEEE International Conference on Cognitive Infocommunications (CogInfoCom) 2017, 2017(9) pp. 243-246, DOI: 10.1109/CogInfoCom.2017.8268250

[4] Adam Csapo, Arni Kristjansson, Hunor Nagy, Gyorgy Wersenyi: Evaluation of Human-Myo Gesture Control Capabilities in Continuous Search and Select Operations. $7^{\text {th }}$ IEEE International Conference on Cognitive Infocommunications (CogInfoCom). 2016, pp. 415-420, DOI: 10.1109/CogInfoCom.2016.7804585

[5] Gergely Sziladi, Tibor Ujbanyi, Katona, Attila Kovari: The analysis of hand gesture based cursor position control during solve an IT related task, $8^{\text {th }}$ IEEE International Conference on Cognitive Infocommunications, 2017, pp. 413-418

[6] Tibor Ujbanyi, et al.: Eye-Tracking Analysis of Computer Networks Exam Question Besides Different Skilled Groups, $7^{\text {th }}$ IEEE International Conference on Cognitive Infocommunications, 2016, pp. 277-281

[7] Jozsef Katona, et al.: Electroencephalogram-Based Brain-Computer Interface for Internet of Robotic Things, Cognitive Infocommunications, Theory and Applications, 2018, pp. 253-275

[8] Costescu Cristina, et al.: Assessing Visual Attention in Children Using GP3 Eye Tracker, Proceedings of the $10^{\text {th }}$ IEEE International Conference on Cognitive Infocommunications, 2019, pp. 343-348

[9] Anna Sudár, Ádám B. Csapó: Interaction Patterns of Spatial Navigation in VR Workspaces, Proceedings of the $10^{\text {th }}$ IEEE International Conference on Cognitive Infocommunications, 2019, pp. 611-614

[10] Z. Kvasznicza: Teaching electrical machines in a 3D virtual space, $8^{\text {th }}$ IEEE International Conference on Cognitive Infocommunications, Debrecen, 2017, pp. 385-388

[12] Ilona Heldal, Carsten Helgesen: The Digital HealthLab: Supporting Interdisciplinary Projects in Engineering and in Health Education, Journal of Applied Technical and Educational Sciences, Vol. 8, No. 4, 2018, pp. 421

[13] Laszlo Bognar, Eva Fancsikne, Peter Horvath, Antal Joos, Balint Nagy, Gyorgyi Strauber: Improved learning environment for calculus courses, 
Journal of Applied Technical and Educational Sciences, Vol. 8, No. 4, 2018, pp. 35-43

[14] Csaba Rigóczki, Andrei Damsa, Kristóf Györgyi-Ambró: Gamification on the edge of educational sciences and pedagogical methodologies, Journal of Applied Technical and Educational Sciences, Vol. 7, No. 4, 2017, pp. 79-88

[15] Attila Kovari: CogInfoCom Supported Education: A review of CogInfoCom based conference papers, Proceedings of the $9^{\text {th }}$ IEEE International Conference on Cognitive Infocommunications (CogInfoCom), 2018, pp. 233-236

[16] Robert Pinter, Sanja Maravic Cisar: Measuring Team Member Performance in Project Based Learning, Journal of Applied Technical and Educational Sciences, Vol. 8, No. 4, 2018, pp. 22-34

[17] Hungarian Stroke Society: Heart disease and stroke statistics: report from the Hungarian Stroke Society. Hungarian Journal of Stroke, 2011(9)

[18] Emilia Benjamin, Michael J Blaha, Stephanie E Chiuve, Mary Cushman: Heart disease and stroke statistics 2017 update: a report from the American Heart Association. Circulation, Vol. 135, 2017, pp. 229-445

[19] Quanhe Yang, Xin Tong, Linda Schieb, Adam Vaughan: Recent trends in stroke death rates - United States, 2000-2015, National Center for Chronic Disease Prevention and Health Promotion Division for Heart Disease and Stroke Prevention, MMWR Morbidity and mortality weekly report. 2017, 66(35) DOI: 10.15585/mmwr.mm6635e1

[20] Pablo Arias, Veronica Robles-García, Gabriel Sanmartín, Julian Flores, Javier Cudeiro: Virtual reality as a tool for evaluation of repetitive rhythmic movements in the elderly and Parkinson's disease patients. PloSOne, 2012, Vol. 7, e30021

[21] Stephanie M. N. Glegg, Danielle E. Levac: Barriers, Facilitators and Interventions to Support Virtual Reality Implementation in Rehabilitation: A Scoping Review. PM\&R, Vol. 10, 2018, pp. 1237-1251, DOI:10.1016/j.pmrj.2018.07.004

[22] Ian Davidson, Karen Waters: Physiotherapists Working with Stroke Patients. Physiotherapy, Vol. 86, 2000, pp. 69-80, DOI:10.1016/s00319406(05)61208-4

[23] Ozgur Zeliha Karaahmet, Eda Gurcay, Ozlem C Avluk, Eda Umay, Ibrahim Gundogdu, Oznur Ecerkale, Aytul Cakci: Poststroke depression: Risk factors and potential effects on functional recovery. International Journal of rehabilitation research. Internationale Zeitschrift fur Rehabilitationsforschung. Revue Internationale de Recherches de readaptation. $\quad$ Vol. $40(1) \quad 2017, \quad$ pp. 71-75, DOI:10.1097/MRR.0000000000000210 
[24] Kelly J. Bower, Julie Louie, Yoseph Landesrocha, Paul Seedy, Alexandra Gorelik, Julie Bernhardt: Clinical feasibility of interactive motioncontrolled games for stroke rehabilitation. Journal of NeuroEngineering and Rehabilitation, Vol. 11, 2015, pp. 12-63, DOI:10.1186/s12984-015-0057-x

[25] David Webster, Oskar Celik: Systematic review of Kinect applications in elderly care and stroke rehabilitation. Journal of NeuroEngineering and Rehabilitation, Vol. 11, 2014, pp. 108-118, DOI:10.1097/MRR.0000000000000210

[26] Bruno Bonnechère, Bart Jansen, Lubos Omelina, Jan Van Sint et al.: The use of commercial video games in rehabilitation: a systematic review. International Journal of rehabilitation research. Vol. 39, 2016, pp. 277-290

[27] Nadia Hocine, Abdelkader Gouaïch, Stefano A. Cerri, Denis Mottet, Jérôme Froger, Isabelle Laffont: Adaptation in serious games for upperlimb rehabilitation: an approach to improve training outcomes. User Modeling and User-Adapted Interaction, Vol. 25, 2015, pp. 65-98, DOI:10.1007/s11257-015-9154-6

[28] Mark A. Livingston, Jay Sebastian, Zhuming Ai, Jonathan W. Decker: Performance measurements for the Microsoft Kinect skeleton. NAVAL RESEARCH LAB WASHINGTON DC. Vol. (3) 2012, pp. 119-120, DOI: 10.1109/VR.2012.6180911

[29] Oliver Wasenmüller, Didier Stricker: Comparison of Kinect v1 and v2 depth images in terms of accuracy and precision. Asian Conference on Computer Vision, 2016(11) pp. 34-45

[30] Simeon Keates, Pat Langdon, P. John Clarkson, Peter Robinson: User Models and User Physical Capability. User Modeling and User-Adapted Interaction, Vol. 12(2) 2002 pp. 139-169, DOI:110.1023/A:1015047002796

[31] Roberto Llorens, Enrique Noé, Carolina Colomer, Mariano Luis Alcaniz: Effectiveness, Usability, and Cost-Benefit of a Virtual Reality-Based Telerehabilitation Program for Balance Recovery After Stroke: A Randomized Controlled Trial. Archives of Physical Medicine and Rehabilitation, Vol. 96, 2015, pp. 418-425, DOI:10.1016/j.apmr.2014.10.019

[32] Lise Worthen-Chaudhari: Effectiveness, Usability, and Cost-Benefit of a Virtual Reality-Based Telerehabilitation Program for Balance Recovery After Stroke: A Randomized Controlled Trial. Archives of Physical Medicine and Rehabilitation, Vol. 96, 2015, pp. 1544, DOI:10.1016/j.apmr.2015.03.025

[33] Imad Afyouni, Faizan Ur Rehman, Ahmad Muaz Qamar, Soahib Ghani, Syed Osama Hussain, Bilal Sadiq, Saleh Basalamah: A therapy-driven gamification framework for hand rehabilitation. User Modeling and User- 
Adapted Interaction, Vol. 27, 2017, pp. 215-265, DOI:10.1007/s11257-0179191-4

[34] Arin Ghazarian, Majid Noorhosseini: Automatic detection of users' skill levels using high-frequency user interface events. User Modeling and UserAdapted Interaction, Vol. 20(2), 2010, pp. 109-146, DOI:10.1007/s11257010-9073-5

[35] Francis Quek: Toward a Vision-Based Hand Gesture Interface. Virtual Reality Software and Technology. 1994(8) DOI: 10.1142/9789814350938_0003

[36] Vladimir I. Pavlovic, Rajeev Sharma, Thomas S. Huang: Visual Interpretation of Hand Gestures for Human-Computer Interaction: A Review. IEEE TRANSACTIONS ON PATTERN ANALYSIS AND MACHINE INTELLIGENCE, Vol. 19, 1997, pp. 677-695 